\title{
Clinicopathological Profile of Clear Cell Renal Cell Carcinoma
}

\author{
Pamela Kusumadewi Putri Thaib \\ Department of Anatomical Pathology, Faculty of Medicine, Universitas Airlangga, Surabaya, Indonesia | \\ Universitas Airlangga Academic Hospital, Surabaya, Indonesia |Dr. Soetomo General Academic Hospital, \\ Surabaya, Indonesia \\ Email: pamela.thaib@gmail.com \\ Anny Setijo Rahaju \\ Department of Anatomical Pathology, Faculty of Medicine, Universitas Airlangga, Surabaya, Indonesia | \\ Universitas Airlangga Academic Hospital, Surabaya, Indonesia |Dr. Soetomo General Academic Hospital, \\ Surabaya, Indonesia \\ Corresponding author email: anny_sr@fk.unair.ac.id
}

\begin{abstract}
Clear cell renal cell carcinoma (ccRCC) is the most common subtype of kidney cancer with aggressive behavior. Tumor stage and grade are important parameter in diagnosis. Data about the clinical and pathology profile of ccRCC in Indonesia is limited, therefore further information is needed. The study aimed to determine clinicopathologic profile of ccRCC in Soetomo Academic General Hospital (SAGH). This descriptive study was conducted retrospectively with cross-sectional approach. Clinical and histopathological archives of ccRCC specimen were retrieved during January 2016 to December 2020. The total number of ccRCC cases obtained was 38 cases, the most cases (11) occurred in 2016. The average of patient's age was 55.64 years. Patients were predominantly male (71.1\%), with a ratio to female 2.5:1. The tumors mostly filled the entire kidney, in 15 (39.5\%) cases. Most pT stage distribution was pT2, in 15 (39.5\%) cases. Most N stage distribution was NO, in 23 (60.5\%) cases. Only $5(13.2 \%)$ cases showed multiple pulmonary nodules which suspected to be metastatic (M1). The most common tumors grade was G4, in 17 (44.7\%) cases. In conclusion, ccRCC patient were predominantly male, tumors mostly high grade, filled entire kidney, not penetrated renal capsule, and not metastasize to regional lymph nodes.

Keywords---carcinoma, clinicopathological, grade, incidence, kidney cancer
\end{abstract}

\section{Introduction}

Renal cell carcinoma is a malignant neoplasm originated from renal epithelial cells and constitute more than $90 \%$ of malignant neoplasms in the kidney. This carcinoma includes more than 10 histological subtypes, with clear cell renal cell carcinoma (ccRCC) being the most common type (80\%), aggressive, and the most common cause of death because its high propensity of metastasis to bone, lung, and liver (Hakimi et al., 2016; Moch et al., 2016; HsiehJJ et al., 2017; Padala et al., 2020). The synonym for this neoplasm is Grawitz tumour or hypernephroma (Moch et al., 2016; HsiehJJ et al., 2017). GLOBOCAN data in 2020 showed that 323,091 people/year were diagnosed with kidney cancer worldwide, occupying the 14th most common cancer incidence worldwide and the 10th most common in developed countries. Men are more affected than women with a ratio about 2:1 (Padala et al., 2020). The peak incidence of this malignancy is the age of 60--years (Bergstrom et al., 2001; Padala et al., 2020). The overall incidence of kidney cancer in Indonesia is around 2.4-3 cases in 100,000 population (Ferlay et al., 2013).

The majority of these cancers occurred sporadically, only minority were hereditary. Inactivation of the von Hippel- Lindau (VHL) gene underlies the development of ccRCC tumour cells, both in sporadic and hereditary cases (Brugarolas, 2014). Altered fatty acids and glucose metabolism is occurred during the development these tumour cells, so that the morphological characteristics of ccRCC are rich in lipid and glycogen content, with high vascularity (Hakimi et al., 2016). A clear to mild eosinophilic cytoplasm, thus giving a clear cell appearance, was resulted from high lipid and glucose content in the cytoplasm of tumour cells that were lysed during the histopathological process 
(Campbell \& Rini, 2009). The progress of this tumour can be assessed from several aspects, including TNM stage and tumour grade.

Tumors stage is the most important parameter affecting the survival of ccRCC patients, followed by tumour grade (Erdogan et al., 2004; Taneja \& Williamson, 2018). Renal cell carcinoma staging refers to the WHO (World Health Organization) Classification Tumours of the Urinary System and Male Genital Organ / 8th edition of TNM AJCC (American Joint Committee on Cancer) system, which is generally based on local extension of the primary tumour and invasion into adjacent tissue $(\mathrm{T})$, regional lymph node involvement $(\mathrm{N})$, and the presence of distant metastases (Rini, 2017). The pathological stage of TNM in anatomical pathology examination can be assessed if the tissue sample is received and proven microscopically by histopathological examination, while the clinical stage of TNM can be assessed from the pre-operative CT-scan or MRI imaging results (Moch et al., 2016; Taneja \& Williamson, 2018; Hamid et al., 2019). An increase in T, N, and M stages is associated with worse survival, recurrence, and prognosis (Taneja \& Williamson, 2018). Increasing tumour grade is also associated with worse survival rates and prognosis (Moch et al., 2016; Taneja \& Williamson, 2018).

The classification of pathological T staging (pT) is divided into 4 stages which assesses the primary tumour for both size and invasion into adjacent tissue, namely pT1 (tumors greatest dimension is less than $7 \mathrm{~cm}$ and still limited in the kidney; pT1a: tumour $4 \mathrm{~cm}$ or less; pT1b: tumour more than $4 \mathrm{~cm}$ but not more than $7 \mathrm{~cm}$ ), pT2 (tumour greatest dimension is more than $7 \mathrm{~cm}$ and still limited to the kidney; pT2a: tumour more than $7 \mathrm{~cm}$ but not more than $10 \mathrm{~cm}$; pT2b: tumour more than $10 \mathrm{~cm}$ ), pT3 (tumour grows beyond renal capsule into the perirenal tissue or into the renal vein, renal sinus, segmental vein, inferior cava vein but has not penetrated the Gerota fascia; pT3a: tumour grossly extends into renal vein or its segmental branches or invades perirenal and/or sinus fat; pT3b: tumour grossly extends into the vena cava below diaphragm; pT3c: tumour grossly extends into the vena cava above the diaphragm or invades the wall of the vena cava) and pT4 (tumour invades the Gerota fascia and/or ipsilateral adrenal gland) (Moch et al., 2016; Taneja \& Williamson, 2018).

Regional lymph nodes metastases or stage $\mathrm{N}$ is divided into 2 stages, stages $\mathrm{N} 0$ (no regional lymph node metastases) and N1 (regional lymph node metastases found). Stage M, which is the absence or presence of tumour cell metastases in distant organs is divided into stages M0 (no distant metastases) and M1 (distant metastases found). Tumour grade $(\mathrm{G})$ is classified based on the differentiation of the nucleus or nucleolus of tumour cells, divided into G1 (absent, basophilic nucleoli at 400x magnification), G2 (prominent nucleoli at 400x magnification only, but not visible at 100x magnification), G3 (prominent nucleoli at 100x magnification), and G4 (extreme nuclear pleomorphism, multinucleated giant cells, sarcomatoid or rhabdoid differentiation) (Moch et al., 2016; Taneja \& Williamson, 2018).

The diagnosis of ccRCC may be directed by clinical and radiological examinations, but anatomical pathology examination is essential to determine a definitive diagnosis, including TNM stage and tumors grade (Moch et al., 2016; Taneja \& Williamson, 2018). Morphological identification of ccRCC through routine histopathological examination and followed by immunohistochemical examination if needed, is very important in order to provide a correct diagnosis so that the management and therapeutic strategy of this tumors can be more accurate and targeted (Moch et al., 2016; Taneja \& Williamson, 2018). This retrospective study was conducted due to the scarcity of studies concerning the clinicopathological profile of ccRCC tumours in Indonesia and to provide insight about these neoplasms in a referral hospital in East Java, Indonesia (Jayson \& Sanders, 1998; Wang \& Gaz, 1985).

\section{Material and Method}

This descriptive observational study was conducted on archived formalin-fixed paraffin-embedded tissue samples and histopathological H\&E slides obtained from 38 patients who underwent nephrectomy and diagnosed as clear cell renal cell carcinoma in Anatomical Pathology Laboratory of Dr. Soetomo General Academic Hospital (SGAH), Surabaya, Indonesia, throughout January 2016 until December 2020. The clinical parameter comprising patient's gender, age, regional lymph node imaging result, and distant metastatic specific organ imaging result were obtained from medical record archive. Medical records were also reviewed and documented regarding the data of tumour size and tumour extension into renal capsule, perirenal fat, renal sinus, renal pelvis, major renal vein, Gerota fascia, also ipsilateral adrenal gland. The pathological parameter in this study were tumour location, $\mathrm{T}$ stage and tumour grade. All status of regional lymph node and distant metastatic organs involvement were classified into N stage (N0, N1) and M stage (M0, M1), respectively. All of the H\&E-stained slides from each case were reviewed and classified according to T stage (pT1, pT2, pT3, pT4) and tumour grade (G1, G2, G3, G4) (Irwin, 2016; Williams \& Lees, 2009). 
Results

The total number of ccRCC cases within 5 years was 38 cases, after applied inclusion criteria. Previous excluded samples were 3 cases, consist of 1 case which after reviewing the histopathological H\&E slide was clear cell papillary renal cell carcinoma, 1 case of differential diagnosis of ccRCC with sarcomatoid differentiation but after immunohistochemical examination it was an inflammatory myofibroblastic tumor, and 1 case with incomplete paraffin block and histopathological slide. The most cases were in 2016 with 11 cases, followed by 2018, 2020, 2017, and 2019 with 10, 7, 7, and 3 cases respectively. The patients were ranged from age 29 to 69 years old, with the average of patient's age was $55.64 \pm 5.13$ years. The highest age group was $41-50,51-60$, and 61-70 interval, amount for 12 (31.6\%) patients equally. The least age group was 21-30 years, counted for 2 (5.2\%) patients. No case found below 20 years neither above 70 years. Male sex was predominant, counted for $27(71.1 \%)$ cases, and with a ratio to female was $2.5: 1$.

The location of the tumor in the sample of this study was determined from macroscopic examination at the Anatomical Pathology Laboratory of SGAH. After unilateral nephrectomy specimen was received, a macroscopic evaluation of the kidney was carried out, one of which was the orientation of the tumor location. The location of the tumor in the kidney was divided into 5 groups, namely the superior pole, medial pole, inferior pole, multifocal tumor, and the tumor filled the entire kidney. The most common tumors was the tumors that filled the entire kidney counted for $15(39.5 \%)$ cases, followed by tumors in the medial pole in $14(36.8 \%)$ cases, superior pole in $5(13.2 \%)$ cases, multifocal tumors in $3(7.9 \%)$ cases, and inferior pole in $1(2.6 \%)$ case. One case with multifocal tumors was found in a patient aged 29 years (Buckner, 2003; Guyatt et al., 2011).

The highest distribution of T stage was stage pT2, as many as $21(48.8 \%)$ cases, followed by stage pT3, pT1, and at least stage pT4, counted for $4(10.5 \%)$ cases. Two cases of stage pT4 ccRCC tumors showed tumors invasion into the ipsilateral adrenal gland and other 2 cases showed tumors penetration beyond the Gerota fascia. This study assessed stage $\mathrm{N}$ not based on histopathological examination but based on the results of routine preoperative CT-scan and/or MRI imaging, which evaluated the presence or absence of regional lymphadenopathy, therefore included in the clinical N stage. The distribution of N0 stage was majority in this study as many as $23(60.5 \%)$ cases and N1 was the minority. Paraaortic or parailiac lymphadenopathy with suspected metastases was discovered in all stage N1 cases. Pre-operative CT imaging, MRI, and/or bone survey examination, which assess suspicious metastatic nodules in distant organs, were not examined in all patients. The majority of the patients in this study were only examined for CT-scan and/or MRI of the abdomen. Suspected tumors metastases in the lungs were found in 5 (13.2\%) patients in this study, which on CT-scan and/or thoracic MRI showed multiple pulmonary nodules. Therefore, TNM staging cannot be established because there were no complete data about $\mathrm{M}$ stage in this ccRCC cases. The most common tumour grade in this study was grade 4 with $17(44.7 \%)$ cases, followed by grade 3 with $10(26.3 \%)$ cases, grade 2 with $8(21.1 \%)$ cases, and at least grade 1 with $3(7.9 \%)$ cases. The clinicopathological characteristics of the samples are shown in Table 1. The microscopic features of various $\mathrm{T}$ stages and tumors grades are presented in Figure 1 and Figure 2, respectively.

Table 1

Characteristic of clinicopathological profile

\begin{tabular}{lll}
\hline & Characteristics & \multicolumn{1}{c}{$\mathrm{n}(\%)$} \\
\hline \multirow{4}{*}{ Year } & 2016 & $11(28.9)$ \\
& 2017 & $7(18.4)$ \\
& 2018 & $10(26.4)$ \\
& 2019 & $3(7.9)$ \\
Age & 2020 & $7(18.4)$ \\
& $21-30$ years & $2(5.2)$ \\
& 31-40 years & $0(0)$ \\
Sex & 41-50 years & $12(31.6)$ \\
& 51-60 years & $12(31.6)$ \\
& 61-70 years & $12(31.6)$ \\
Tumor & Male & $27(71.1)$ \\
& Female & $11(28.9)$ \\
& Superior pole & $5(13.2)$ \\
& Medial pole & $14(36.8)$ \\
& Inferior pole & $1(2.6)$
\end{tabular}


location

\begin{tabular}{lll} 
& Multifocal & $3(7.9)$ \\
& Entire kidney & $15(39.5)$ \\
& pT1 & $6(15.8)$ \\
T stage & pT2 & $15(39.5)$ \\
& pT3 & $13(34.2)$ \\
& pT4 & $4(10.5)$ \\
N stage & N0 & $23(60.5)$ \\
& N1 & $15(39.5)$ \\
M stage & Mx & $33(86.8)$ \\
& M0 & $n / a^{\mathrm{b}}$ \\
& M1 & $5(13.2)$ \\
Tumor grade & G1 & $3(7.9)$ \\
& G2 & $8(21.1)$ \\
& G4 & $10(26.3)$ \\
\hline
\end{tabular}
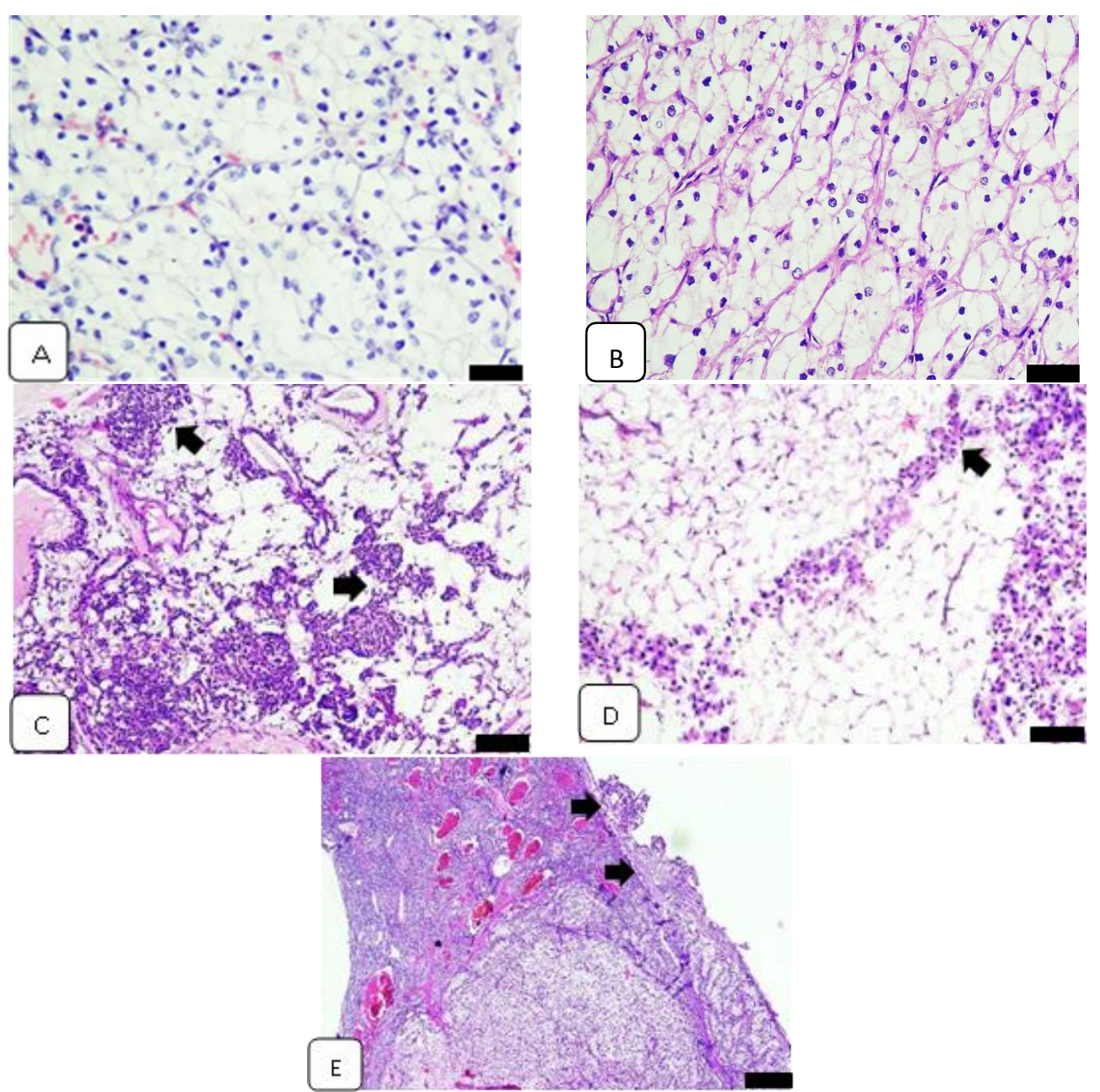

Figure 1. Photomicrograph of pT1-pT4 stage of clear cell renal cell carcinoma (H\&E staining). A). pT1; B). pT2 (A \& B: 400x magnification); C-D). pT3a, tumour cells invasion into perirenal fat (black arrow; 100x and 200x

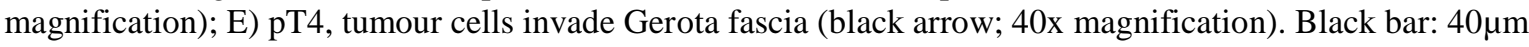



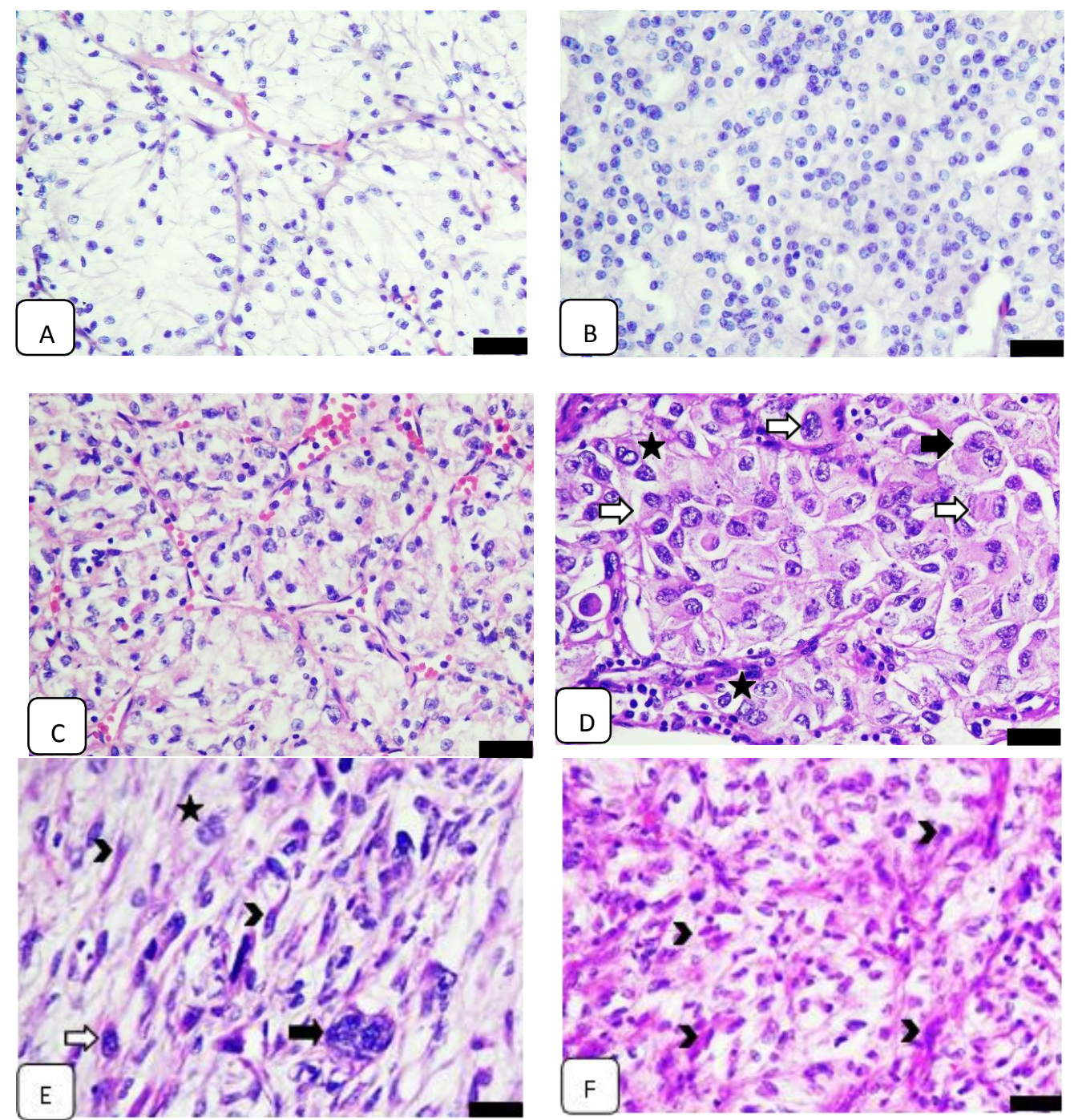

Figure 2. Photomicrograph of tumour grade 1-4 of clear cell renal cell carcinoma (H\&E staining). A). G1; B). G2; C). G3; D). G4, pleomorphic (star), sarcomatoid (rhabdoid, white arrow); E). G4, pleomorphic (star), sarcomatoid (rhabdoid, white arrow), multinucleated giant cells (black arrow); F). G4, sarcomatoid (spindle, arrowhead). A-F: 400x magnification. Black bar: $40 \mu \mathrm{m}$

\section{Discussion}

This 5 years retrospective study exhibited 38 patients of clear cell renal cell carcinoma. The highest number of ccRCC patients was found in 2016 with 11 (28.9\%) cases. The number of these cases varies greatly per year. The number of cases in 2019 was obtained at least ( 3 cases) due to a decrease in the number of oncology patients visiting hospital that year due to an outbreak of the COVID-19 pandemic. The average patient's age was 55.64 years, this result slightly closer to previous study in Indonesia which found the highest age range of kidney cancer was between 51-65 years group Hamid et al. (2019), but younger than the previous study in America which obtained 64 years (Padala et al., 2020). Male patients were predominant (69.8\%) in this study with a ratio male to female was 2.5:1. This finding is in line with earlier investigations (Moch et al., 2016; Hamid et al., 2019; Padala et al., 2020). This may be caused by some risk factors in men such as excessive alcohol consumption, smoking habits, hypertension, and occupational exposure to trichloroethylene industrial materials (Fowkes et al., 2003; Kim et al., 1995).

The most common locations for ccRCC tumours in kidney were tumours that filled the entire kidney in 15 $(39.5 \%)$ cases, followed by tumours on the medial pole, superior pole, multifocal tumours, and inferior pole. Tumours of ccRCC are usually solitary cortical tumours that can occur in both kidneys. Tumours that fill the entire kidney will increase the chances of higher tumour stages, tumour dissemination to the lymph nodes, tumour 
extension to the adrenal glands and distant metastases (Sagalowsky et al., 1994; Sandock et al., 1997; Taneja \& Williamson, 2018; Padala et al., 2020). Tumours on the medial pole of kidney should be sampled more carefully between the renal pelvicalyceal area and renal parenchyma, which contains the main lymphovascular supply of kidney. In this medial area, pathologist must evaluate whether there is tumour invasion into the renal sinus, renal vein, segmental vein, or perirenal adipose tissue, which crucial in $\mathrm{T}$ staging, a determinant pathway for metastasis, and an important factor for prognosis (Taneja \& Williamson, 2018).

Tumours of ccRCC on superior pole will increase the chance of tumour dissemination to adrenal glands, which will escalate tumour progression to stage T4 (Sagalowsky et al., 1994; Sandock et al., 1997). The adrenal glands tend to be preserved if there is no suspicion of being invaded by the tumour, but if the tumour is located on the upper pole of the kidney and appeared abnormal then adrenalectomy may be an option (HsiehJJ et al., 2017; Hamid et al., 2019). Tumours located on the superior pole of kidney and a nephrectomy specimen involving resection of the ipsilateral adrenal gland should be evaluated and carefully sampled by a pathologist. Multifocal and/or bilateral tumours which occurred in less than $5 \%$ of cases are usually typical of hereditary cancer syndromes such as von Hippel-Lindau syndrome (Moch et al., 2016). This study did not examine the mutation of VHL gene, however, 1 case with a multifocal tumour found in 29 years patient. The author assumed that this patient might have a VHL syndrome.

Various somatic mutations of VHL have been found in ccRCC which may be occurred sporadically or hereditary. In normal condition, VHL protein has the capacity to degrade HIF (Octavianda \& Rahaju, 2018). This loss of function of von Hippel-Lindau protein appears to play a role in tumour initiation, progression, and metastasis (Lonser et al., 2003; Klatte et al., 2009; Moch et al., 2016). The mutation of VHL protein will cause HIF $\alpha$ (hypoxiainducible factor) cannot be hydroxylated so that the VHL complex cannot targeting HIF $\alpha$ for degradation, which will cause HIF1 $\alpha$ (HIF1A) and HIF2 $\alpha 12$ (EPAS1) accumulation, even in the adequate oxygenated tumour microenvironment (Majmundar et al., 2010). Decreased cellular oxygen concentration will increase HIF1 $\alpha$ activity. HIF $1 \alpha$ activates large gen energy reserve to increase oxygen availability or provide metabolic adaptation in cell hypoxia conditions (Zagzag et al., 2005). The first pathway of HIF is a transcription factor that regulates various hypoxia-associated target genes transcription such as vascular endothelial growth factor (VEGF), platelet-derived growth factor polypeptide (PDGF $\beta$ ), erythropoietin, transforming growth factor (TGF $\alpha$ ), glucose transporter 1 (GLUT1), and also the CXCR4/CXCL12 axis. HIF2 $\alpha$, like HIF1 $\alpha$, will dimerize with HIF1 $\beta$ and its expression is increased upon pVHL inactivation (Zagzag et al., 2005).

The second pathway of HIF $\alpha$ will disintegrate the extracellular matrix through the activity of matrix metalloproteinase-2 (MMP-2), which promotes tumour invasion and angiogenesis via the activation of VEGF. The outcome may represent as an invasive tumour with high vascularity (Kurban et al., 2006). One study theorized that loss of function of the VHL gene alone was not sufficient to induce the development of ccRCC, but additional genetic and/or epigenetic events may be required for tumour cell development (Kaelin, 2007). Mutations in the PBRM1, SETD2, BAP1, KDM5C and MTOR genes were found in ccRCC, which were associated with disease progression in the presence of inactivation of the VHL gene (Brugarolas, 2014). Other cytogenetic abnormalities associated with ccRCC were addition of $5 q$ allele $(33 \%)$, loss of $14 q$ allele chromosome (28\%), trisomy 7 (26\%), loss of the $8 p$ allele (20\%), 6q (17\%), 9p (16\%), 4p (13\%) and loss of $Y$ chromosome in males (55\%). Tumours with loss of $3 p$ allele exhibited a lower TNM stage, thus providing a better prognosis. Loss of $4 p, 9 p$, and 14q alleles were associated with higher TNM staging, higher grade, and larger tumour size, thus conferring a poorer prognosis (Klatte et al., 2009). Deletion of chromosome 14q was associated with HIF1A, which predicted lead to more aggressive disease ( Campbell \& Rini, 2009; Cancer Genome Atlas Research Network, 2013).

Assessing tumour stage in ccRCC is very important because it can provide prognostic information, predict survival, metastasis and disease recurrence, as well as its main function as the basis for disease management. Clinical staging is important in determining treatment options, such as ablation therapy, radical nephrectomy, or partial nephrectomy. Pathological staging is a useful tool for determining subsequent postoperative therapy and to schedule clinical follow- up also consultation. The distribution of patients was found the most in pT2 group with 15 (39.5\%) cases, followed by pT3 group, the pT1 group, and at least pT4 group. Two cases in pT4 group showed direct tumour extension to the ipsilateral adrenal gland and two cases had tumour invasion beyond Gerota's fascia. The classic symptoms of kidney cancer such as hematuria and lower back pain (flank area) were likely encouraged patients to seek medical treatment. Local stage group, a combination of pT1 and pT2 cases, was counted for $55.3 \%$ cases in this study, but the result was slightly different from the Surveillance, Epidemiology, and End Results (SEER) data which showed that most kidney cancer patients present with local stages $(65 \%)$ (Taneja \& Williamson, 2018). That difference between the result in developed countries and Indonesia may be caused by delayed visit to the hospital, which may due to lack of patient awareness, afraid of treatment, limited costs or perhaps limited patient's access 
from remote area to acquire an optimal treatment. This study exhibited only a few cases of pT1 (15.8\%) and some patients with stage pT4 were still found, which may due to comprehensive medical check-up and radiological imaging of kidney as an early detection of kidney cancer are not a routine examination (Vogelzang \& Stadler, 1998; Jewett et al., 2011).

This study assessed the status of $\mathrm{N}$ stage, the presence or absence of regional lymph node metastases, not based on the result of histopathological examination of regional lymph nodes, but based on CT-scan and/or MRI imaging which is a routine preoperative examination to assess regional lymphadenopathy. Imaging findings that exhibited para-aortic or para-iliac lymphadenopathy with suspicious dissemination of tumour cells were classified as N1. Some literatures stated that retroperitoneal lymph node dissection (RLPND) procedure in kidney cancer did not show a significant benefit and difference in cancer-specific mortality, overall survival, and progression-free-survival (Blom et al., 2009; Gershman et al., 2017; Dispagna et al., 2021). Regional lymph node dissection was often performed in young patients and advanced disease (Taneja \& Williamson, 2018). Pathologist has to palpate and dissect especially around the hilar area to evaluate lymph nodes in all nephrectomy specimens, however, random adipose tissue sampling without visible macroscopic lymph node is unnecessary because of the low probability of obtaining lymph nodes with microscopically tumour cell infiltration (Taneja \& Williamson, 2018).

The most $\mathrm{N}$ stage distribution of this study was N0 in 23 (60.5\%) cases and stage N1 in 15 (39.5\%) cases. The microscopic assessment of tumour cell metastases in regional lymph nodes cannot be carried out without lymph node dissection specimen, so the classification of $\mathrm{N}$ stage in this study was carried out based on the results of radiological imaging. The presence of tumour cell metastases in regional lymph nodes will increase ccRCC stage and tumour cells dissemination to metastatic specific organs. Several studies found that the presence of regional lymph node metastases was a very important prognostic factor in renal cell carcinoma (Klatte et al., 2018). The 5-year survival rate of renal cell carcinoma was $92.6 \%$ for locally grown tumours, decreasing in regional lymph node spread (66.7\%) and metastasis (11.7\%) (Taneja \& Williamson, 2018). Surveillance, Epidemiology, and End Results (SEER) data showed that kidney cancer patients present with lymph node metastases was counted for $16 \%$ Taneja \& Williamson (2018), while in this study the prevalence of stage N1 cases was more than $30 \%$. Same as the T stage status, this may be caused by delayed visit to the hospital, which may due to lack of awareness, afraid of treatment, limited costs or limited patient's access to reach the hospital (Dharmayuda et al., 2021; Arimbawa et al., 2021).

Chest and head CT-scan, MRI, and/or bone survey which assess the presence or absence of suspicious nodules in distant organs, were not examined in all this study sample. The majority of the sample in this study were only examined for CT-scan and/or MRI of the abdomen. Suspicion of tumour metastases in the lungs was found in 5 $(13.2 \%)$ cases in this study, which on thoracic CT-scan and/or MRI showed multiple pulmonary nodules suspicious for tumour metastases. The presence of M1 stage in ccRCC patients regardless of T stage or N stage will increase the patient's status to advanced and end-stage disease. The author considered that a thorough and comprehensive radiological examination of specific metastatic site was not routinely performed in all cases of kidney cancer of there were no clinical sign of metastasis. Therefore, the TNM stage cannot be assessed in this study.

The most common tumour grade in this study was grade 4 with 17 (44.7\%) cases, followed by grade 3, grade 2, and at least grade 1. Tumour grade should always be assessed and included in the anatomical pathology report because the grading system is validated as a prognostic indicator for ccRCC. Tumours with grade 4 showed a worse prognosis than grade 1, especially if sarcomatoid features were present (Moch et al., 2016; Hamid et al., 2019). Renal cell carcinoma with sarcomatoid or rhabdoid differentiation, which is a grade 4, has poor prognosis with a median survival time of 5.9-19 months after diagnosis and a 5-year specific survival was 6\%-22\% (Mikami et al., 2011). de Peralta- Venturina et al. found that sarcomatoid differentiation was found in $8 \%$ ccRCC, which could resemble fibrosarcoma, malignant fibrous histiocytoma, or undifferentiated sarcoma. This sarcomatoid differentiation showed a poorer prognosis where in $69 \%$ of patient's death was related to the cancer, median survival time was 19 months, disease- specific survival rate was $22 \%$ after 5 years, compared with no sarcomatoid differentiation which may live $79 \%$ longer (de Peralta-Venturina et al., 2001). Mesenchymal differentiation of kidney cancer, which has higher propensity to metastasize, is indicated by the epithelial to mesenchymal transition (EMT) process (Chen et al., 2014; Hu et al., 2014).

EMT is a process where epithelial cells lose their intercellular polarity and converted to a mesenchymal phenotype (Chen et al., 2014; Hu et al., 2014; Landolt et al., 2017). This EMT process will increase the potential for tumour migration, invasive behavior, apoptotic resistance, and degradation of extracellular matrix components. The hallmark of EMT is "cadherin switch", i.e. loss of E-cadherin expression, replaced by increased N-cadherin, loss of $\beta$-catenin expression in the cell cytoplasm, and increased $\beta$-catenin expression in the nucleus. $\mathrm{N}$-cadherin, which normally found in mesenchymal cells, was found to be highly expressed in sarcomatoid component of renal cell carcinoma. This cadherin switch process is found in sarcomatoid component of many carcinomas. Cell hypoxia is a common finding in ccRCC due to pVHL inactivation. The hypoxic microenvironment of ccRCC is an important 
factor in promoting the pathological process of EMT, which is a key link in cancer cell progression. The effect of hypoxia was generally mediated by HIF, which was activated by oncogenic signaling mechanisms including the MAPK/RAS and PI3K/AKT pathways (Jiang et al., 2011). EMT transcription factors are zinc-finger transcriptional factors which include Snail (Snail1), Slug (Snail2), Twist, Zeb1, Zeb2, SIP1, and Twist which will induce EMT through E-cadherin repression. Tumour microenvironment under hypoxic conditions will trigger the EMT pathway through the TGF- $\beta$ signaling pathway, Notch, and NF-K $\beta$ signaling (Jiang et al., 2011). Activation of NF-K $\beta$ in the pVHL inactivation condition directly and indirectly induced the expression of Snail, Slug, Twist, Zeb1, and Zeb2 (He \& Magi-Galluzzi, 2014). Exposure to wild-type VHL in ccRCC caused a marked decrease in Snail and SIP1 expression, and was followed by a return of E-cadherin expression (Evans et al., 2007).

\section{Conclusion}

This retrospective ccRCC study identified 38 cases in 5 years period. The most cases were obtained in 2016, the mean age was 55.64 years, predominantly male. The tumour mostly filled the entire kidney. The highest distribution of patients was pT2, N0, and grade 4, with incomplete data regarding M stage. This study may provide knowledge and data about demographic, histopathology, TNM stage and tumour grade of clear cell renal cell carcinoma and may be useful for the basis of therapy and further studies.

\section{Conflict of Interest}

The author declares no conflict of interest within this study.

\section{Ethical Approval}

Ethical approval for this research was issued by Ethics Committee of Dr. Soetomo General Academic Hospital, Surabaya, Indonesia (Reference number: 0482/LOE/301.4.2/VI/2021).

\section{Acknowledgments}

The author expresses the gratitude to the Dean of Faculty of Medicine, Universitas Airlangga and to the President Director of Dr. Soetomo General Academic Hospital for the opportunity given to fulfil the specialist doctor education program and carry out this research, which is a part of the renal cell carcinoma research tree of anatomical pathology research group. The author also expresses the gratitude to all lecturers in Department of Anatomical Pathology, Faculty of Medicine, Universitas Airlangga, Indonesia.

\section{References}

Arimbawa, . I. M., Paramita, A. D. P., Nuaba, I. G. A., \& Saputra, H. (2021). Management of hypocalcemia after modified bilateral radical neck dissection followed with total thyroidectomy: a case report. International Journal of Health Sciences, 5(1), 9-19. https://doi.org/10.29332/ijhs.v5n1.654

Bergström, A., Hsieh, C. C., Lindblad, P., Lu, C. M., Cook, N. R., \& Wolk, A. (2001). Obesity and renal cell cancera quantitative review. British journal of cancer, 85(7), 984-990.

Blom, J. H., van Poppel, H., Maréchal, J. M., Jacqmin, D., Schröder, F. H., de Prijck, L., \& Sylvester, R. (2009). Radical nephrectomy with and without lymph-node dissection: final results of European Organization for Research and Treatment of Cancer (EORTC) randomized phase 3 trial 30881. European urology, 55(1), 28-34.

Brugarolas, J. (2014). Molecular genetics of clear-cell renal cell carcinoma. Journal of clinical oncology, 32(18), 1968.

Buckner, J. C. (2003, December). Factors influencing survival in high-grade gliomas. In Seminars in oncology (Vol. 30, pp. 10-14). WB Saunders. https://doi.org/10.1053/j.seminoncol.2003.11.031

Campbell, S. C., \& Rini, B. I. (2009). Renal cell carcinoma. People's Medical Publishing House USA Limited (PMPH).

Cancer Genome Atlas Research Network. (2013). Comprehensive molecular characterization of clear cell renal cell carcinoma. Nature, 499(7456), 43.

Chen, D., Gassenmaier, M., Maruschke, M., Riesenberg, R., Pohla, H., Stief, C. G., ... \& Buchner, A. (2014). Expression and prognostic significance of a comprehensive epithelial-mesenchymal transition gene set in renal cell carcinoma. The Journal of urology, 191(2), 479-486.

de Peralta-Venturina, M., Moch, H., Amin, M., Tamboli, P., Hailemariam, S., Mihatsch, M., ... \& Amin, M. B. (2001). Sarcomatoid differentiation in renal cell carcinoma: a study of 101 cases. The American journal of surgical pathology, 25(3), 275-284. 
Dharmayuda, T. G., Suega, K., Bakta, I. M., \& Sumohadi, I. M. D. (2021). Ki67 expression and prognostic aspects of colorectal cancer. International Journal of Health Sciences, 5(2), 79-88. https://doi.org/10.29332/ijhs.v5n2.1215

Dispagna, M. A., Daneshvar, M., \& Bratslavsky, G. (2021). Surgical Insights for the Management of Variant Histology in Renal Cell Carcinoma. International braz j urol, 47, 935-942.

Erdoĝan, F., Demirel, A., \& Polat, Ö. (2004). Prognostic significance of morphologic parameters in renal cell carcinoma. International journal of clinical practice, 58(4), 333-336.

Evans, A. J., Russell, R. C., Roche, O., Burry, T. N., Fish, J. E., Chow, V. W., ... \& Ohh, M. (2007). VHL promotes E2 box-dependent E-cadherin transcription by HIF-mediated regulation of SIP1 and snail. Molecular and cellular biology, 27(1), 157-169.

Ferlay, J. S. E. M., Soerjomataram, I., Ervik, M., Dikshit, R., Eser, S., Mathers, C., ... \& Bray, F. (2013). GLOBOCAN 2012 v1. 0. Cancer incidence and mortality worldwide: IARC CancerBase, 11.

Fowkes, F. J. I., Price, J. F., \& Fowkes, F. G. R. (2003). Incidence of diagnosed deep vein thrombosis in the general population: systematic review. European Journal of Vascular and Endovascular Surgery, 25(1), 1-5. https://doi.org/10.1053/ejvs.2002.1778

Gershman, B., Thompson, R. H., Moreira, D. M., Boorjian, S. A., Lohse, C. M., Costello, B. A., ... \& Leibovich, B. C. (2017). Lymph node dissection is not associated with improved survival among patients undergoing cytoreductive nephrectomy for metastatic renal cell carcinoma: a propensity score based analysis. The Journal of urology, 197(3), 574-579.

Guyatt, G. H., Oxman, A. D., Schünemann, H. J., Tugwell, P., \& Knottnerus, A. (2011). GRADE guidelines: a new series of articles in the Journal of Clinical Epidemiology. Journal of clinical epidemiology, 64(4), 380-382. https://doi.org/10.1016/j.jclinepi.2010.09.011

Hakimi, A. A., Reznik, E., Lee, C. H., Creighton, C. J., Brannon, A. R., Luna, A., ... \& Hsieh, J. J. (2016). An integrated metabolic atlas of clear cell renal cell carcinoma. Cancer cell, 29(1), 104-116.

Hamid, A. R. A. H., Umbas, R., Oka, A. A. G., Mochtar, C. A., Djatisoesanto, W., Soedarso, M. A., ... \& Hakim, L. (2019). KANKER GINJAL.

He, H., \& Magi-Galluzzi, C. (2014). Epithelial-to-mesenchymal transition in renal neoplasms. Advances in anatomic pathology, 21(3), 174-180.

HsiehJJ, P., SignorettiS, S., AlbigesL, S., \& HengDY, L. (2017). FicarraV.. Renal cell carcinoma. Nat Rev Dis Primers, 3, 17009.

Hu, T. H., Yao, Y., Yu, S., Han, L. L., Wang, W. J., Guo, H., ... \& Nan, K. J. (2014). SDF-1/CXCR4 promotes epithelial-mesenchymal transition and progression of colorectal cancer by activation of the Wnt/ $\beta$-catenin signaling pathway. Cancer letters, 354(2), 417-426.

Irwin, D. J. (2016). Tauopathies as clinicopathological entities. Parkinsonism \& related disorders, 22, S29-S33. https://doi.org/10.1016/j.parkreldis.2015.09.020

Jayson, M., \& Sanders, H. (1998). Increased incidence of serendipitously discovered renal cell carcinoma. Urology, 5l(2), 203-205. https://doi.org/10.1016/S0090-4295(97)00506-2

Jewett, M. A., Mattar, K., Basiuk, J., Morash, C. G., Pautler, S. E., Siemens, D. R., ... \& Finelli, A. (2011). Active surveillance of small renal masses: progression patterns of early stage kidney cancer. European urology, 60(1), 39-44. https://doi.org/10.1016/j.eururo.2011.03.030

Jiang, J., Tang, Y. L., \& Liang, X. H. (2011). EMT: a new vision of hypoxia promoting cancer progression. Cancer biology \& therapy, 11(8), 714-723.

Kaelin Jr, W. G. (2007). Von hippel-lindau disease. Annu. Rev. Pathol. Mech. Dis., 2, 145-173.

Kim, S. J., Hong, Y. P., Lew, W. J., Yang, S. C., \& Lee, E. G. (1995). Incidence of pulmonary tuberculosis among diabetics. Tubercle and lung disease, 76(6), 529-533. https://doi.org/10.1016/0962-8479(95)90529-4

Klatte, T., Rao, P. N., De Martino, M., LaRochelle, J., Shuch, B., Zomorodian, N., ... \& Pantuck, A. J. (2009). Cytogenetic profile predicts prognosis of patients with clear cell renal cell carcinoma. Journal of clinical oncology, 27(5), 746-753.

Klatte, T., Rossi, S. H., \& Stewart, G. D. (2018). Prognostic factors and prognostic models for renal cell carcinoma: a literature review. World journal of urology, 36(12), 1943-1952.

Kurban, G., Hudon, V., Duplan, E., Ohh, M., \& Pause, A. (2006). Characterization of a von Hippel Lindau pathway involved in extracellular matrix remodeling, cell invasion, and angiogenesis. Cancer research, 66(3), 1313-1319.

Landolt, L., Eikrem, Ø., Strauss, P., Scherer, A., Lovett, D. H., Beisland, C., ... \& Marti, H. P. (2017). Clear cell renal cell carcinoma is linked to epithelial-to-mesenchymal transition and to fibrosis. Physiological reports, 5(11), e13305. 
Lonser, R. R., Glenn, G. M., Walther, M., Chew, E. Y., Libutti, S. K., Linehan, W. M., \& Oldfield, E. H. (2003). von Hippel-Lindau disease. The Lancet, 361(9374), 2059-2067.

Majmundar, A. J., Wong, W. J., \& Simon, M. C. (2010). Hypoxia-inducible factors and the response to hypoxic stress. Molecular cell, 40(2), 294-309.

Mikami, S., Katsube, K. I., Oya, M., Ishida, M., Kosaka, T., Mizuno, R., ... \& Okada, Y. (2011). Expression of Snail and Slug in renal cell carcinoma: E-cadherin repressor Snail is associated with cancer invasion and prognosis. Laboratory investigation, 91(10), 1443-1458.

Moch, H., Cubilla, A. L., Humphrey, P. A., Reuter, V. E., \& Ulbright, T. M. (2016). The 2016 WHO classification of tumours of the urinary system and male genital organs - part A: renal, penile, and testicular tumours. European urology, 70(1), 93-105.

Octavianda, Y., \& Rahaju, A. S. (2018). Increased HIF-1alpha And VEGF Expression Found in Various T Stages of Clear Cell Renal Cell Carcinoma. Folia Medica Indonesiana, 54(2), 102-107.

Padala, S. A., Barsouk, A., Thandra, K. C., Saginala, K., Mohammed, A., Vakiti, A., ... \& Barsouk, A. (2020). Epidemiology of renal cell carcinoma. World journal of oncology, 11(3), 79.

Rini, R. (2017). Fake news and partisan epistemology. Kennedy Institute of Ethics Journal, 27(2), E-43.

Sagalowsky, A. I., Kadesky, K. T., Ewalt, D. M., \& Kennedy, T. J. (1994). Factors influencing adrenal metastasis in renal cell carcinoma. The Journal of urology, 151(5), 1181-1184.

Sandock, D. S., Seftel, A. D., \& Resnick, M. I. (1997). Adrenal metastases from renal cell carcinoma: role of ipsilateral adrenalectomy and definition of stage. Urology, 49(1), 28-31.

Surgical Pathology Clinics, 11(4), pp.797-812. DOI: 10.1016/j.path.2018.07.004

Taneja, K., \& Williamson, S. R. (2018). Updates in pathologic staging and histologic grading of renal cell carcinoma. Surgical pathology clinics, 11(4), 797-812.

Vogelzang, N. J., \& Stadler, W. M. (1998). Kidney cancer. The Lancet, 352(9141), 1691-1696. https://doi.org/10.1016/S0140-6736(98)01041-1

Wang, C. A., \& Gaz, R. D. (1985). Natural history of parathyroid carcinoma: diagnosis, treatment, and results. The American journal of surgery, 149(4), 522-527. https://doi.org/10.1016/S0002-9610(85)80050-7

Williams, D. R., \& Lees, A. J. (2009). Progressive supranuclear palsy: clinicopathological concepts and diagnostic challenges. The Lancet Neurology, 8(3), 270-279. https://doi.org/10.1016/S1474-4422(09)70042-0

Zagzag, D., Krishnamachary, B., Yee, H., Okuyama, H., Chiriboga, L., Ali, M. A., ... \& Semenza, G. L. (2005). Stromal cell-derived factor-1 $\alpha$ and CXCR4 expression in hemangioblastoma and clear cell-renal cell carcinoma: von Hippel-Lindau loss-of-function induces expression of a ligand and its receptor. Cancer research, 65(14), 6178-6188. 